\title{
Differences in Acute Phase Reactants between Gout and Pseudogout
}

\author{
Clement E. Tagoe ${ }^{1 *}$, Yasmin Raza ${ }^{2}$ \\ ${ }^{1}$ Department of Medicine, Albert Einstein College of Medicine, Bronx, USA; ${ }^{2}$ Department of Medicine, Temple University, Phila- \\ delphia, USA.

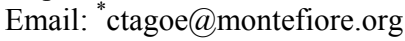

Received October $2^{\text {nd }}, 2013$; revised November $1^{\text {st }}, 2013$; accepted November $25^{\text {th }}, 2013$

Copyright (C) 2013 Clement E. Tagoe, Yasmin Raza. This is an open access article distributed under the Creative Commons Attribution License, which permits unrestricted use, distribution, and reproduction in any medium, provided the original work is properly cited. In accordance of the Creative Commons Attribution License all Copyrights (C) 2013 are reserved for SCIRP and the owner of the intellectual property Clement E. Tagoe, Yasmin Raza. All Copyright (C) 2013 are guarded by law and by SCIRP as a guardian.

\begin{abstract}
Objectives: To define clinical differences in the acute phase response and serum acute phase reactants between gout, pseudogout and crystal-induced arthritis in the presence of non-articular infections (CAI). Patients and Methods: Eleven patients with definite gout, 12 patients with pseudogout and 5 patients with CIA were included in the study. Results: The erythrocyte sedimentation rate (ESR) was significantly different between gout $(68.2 \pm 49.9 \mathrm{~mm} / \mathrm{Hr})$ and CIA $(113.8 \pm 37.2 \mathrm{~mm} / \mathrm{Hr})$ but not between gout and pseudogout $(83.9 \pm 45.6 \mathrm{~mm} / \mathrm{Hr})$ or between pseudogout and CIA. The C-reactive protein (CRP) was significantly increased between gout $(10.1 \pm 7.9 \mathrm{mg} / \mathrm{dL})$ and pseudogout $(18.9 \pm 9.8$ $\mathrm{mg} / \mathrm{dL})$, gout and CIA $(36.5 \pm 12.4 \mathrm{mg} / \mathrm{dL})$ as well as between pseudogout and CIA. The peripheral white cell count was significantly different between gout $(9.27 \pm 3.7 \mathrm{k} / \mu \mathrm{L})$ and CIA $(16.5 \pm 6.8 \mathrm{k} / \mu \mathrm{L})$, and between pseudogout $(8.9 \pm$ $3.2 \mathrm{k} / \mu \mathrm{L}$ ) and CIA. Conclusions: Measurement of ESR and CRP are helpful in crystal-induced arthritis. The CRP has more discriminating utility than the ESR in distinguishing between gout, pseudogout and CIA. Peripheral wbc is most useful for differentiating crystal-induced arthritis from CIA.
\end{abstract}

Keywords: Gout; Pseudogout; Crystal-Induced Arthritis; Acute Phase Reactants; C-Reactive Protein; Calcium Pyrophosphate Dihydrate Deposition Disease

\section{Introduction}

The crystal-induced arthropathies are a group of diseases with a broad range of clinical manifestations from asymptomatic to severely inflammatory. The most common presentations at the inflammatory end of the disease spectrum are gout and pseudogout, precipitated by intraarticular monosodium urate monohydrate (MSUM) and calcium pyrophosphate dihydrate (CPPD) crystals respectively [1]. Both conditions have very high prevalence [2-5]. The resulting health care costs and loss of productivity to society are quite considerable [6].

Gout and pseudogout are highly inflammatory forms of arthritis associated with the release of proinflammatory cytokines through a variety of pathways including the activation of inflammasomes in neutrophils, macrophages and other cell types [7]. However, there are dif-

"Corresponding author. ferences in the way that MSUM and CPPD crystals respectively activate the inflammasome in the quality and quantity of cytokine release. The massive systemic release of inflammatory cytokines causes fever, chills, malaise and the cardinal signs of inflammation locally including tumor, dolor and rubor, which can make the discrimination of crystal-induced arthritis from septic arthritis, cellulitis and other infectious causes difficult to impossible, at least earlier in the presentation. Furthermore, the typical crystal arthropathy patient generally has multiple comorbidities, which adds to the difficulty in establishing a diagnosis and guiding management. Serum acute phase reactants have been suggested as useful tools for refining the diagnosis. It is generally accepted that the erythrocyte sedimentation rate (ESR) and Creactive protein (CRP) are most useful for differentiating infectious from non-infectious causes.

We sought to define the characteristics of gout and 
pseudogout in terms of the acute phase response to see if the ESR and CRP remained useful in discriminating between crystal arthropathy and infection and whether there were any differences in the behavior of those acute phase reactants between gout and pseudogout.

\section{Patients and Methods}

The study was retrospective and included patients admitted to the institution between 2004 and 2012 with definite crystal-induced arthritis from whom there was aspiration of synovial fluid for microbiological, chemical and crystal analysis. Gout was diagnosed using the American college of rheumatology (ACR) diagnostic criteria [8]. Pseudogout was diagnosed by the criteria of McCarty as modified by the European League Against Rheumatism (EULAR) [9,10].

\subsection{Inclusion Criteria}

Eleven subjects with acute gout were included in the study. All gout patients had hyperuricemia and the presence of MSUM crystals in their joint aspirates. Patients were included if they had definite pseudogout with CPPD crystals isolated from the affected joint (10 patients) or if they had probable pseudogout ( 2 patients) in the absence of all of the following; history of gout, persistent hyperuricemia, tophi, radiographic changes suspicious for gout and a non-inflammatory joint aspirate. All patients had negative synovial fluid gram stain and culture. To study the effects of non-articular infections on the serum acute phase reactants five patients with definite gout ( 3 patients) and definite pseudogout ( 2 patients), in the presence of acute infections were included in the study. Hemoglobin levels were $>10 \mathrm{~g} / \mathrm{dL}$ in all patients.

\subsection{Exclusion Criteria}

Patients with acute infections or who had received antibiotics during the hospitalization were excluded from the gout and pseudogout groups. Any subjects with a diagnosis of a well-defined connective tissue disease, positive serum rheumatoid factor, positive serum anti-citrullinated protein antibody (ACPA), positive serum anti-nuclear antibody (ANA), chronic infections such as osteo- myelitis, active HIV infection or active hepatitis were excluded from the study. One patient with joint aspirates showing both MSUM and CPPD crystals was analyzed separately.

Serum acute phase reactants, specifically ESR and CRP obtained within 24 hours of the joint aspiration were considered representative of the gout or pseudogout event. When multiple values were found the results closest to the date and time of aspiration were selected and were invariably the highest values present in such cases. Peripheral white cell count, serum creatinine and serum uric acid were available from the date of joint aspiration as part of the work up for crystal-induced arthritis. Radiographic data from within six months of the admission date were examined for characteristic features of gout or pseudogout including the presence of chondrocalcinosis. The presence of erosions suspicious for gout, in the presence of pseudogout, led to exclusion from the study.

\subsection{Statistical Analysis}

Descriptive statistical analyses using the mean, standard deviation, range and median of variables were employed. Mann-Whitney U-test was used for the pair-wise comparison of means. Group means were compared by oneway Analysis of Variance (ANOVA). Correlations of statistical significance between groups were done using Spearman Rank Correlation. A $P$-value $<0.05$ was considered to be significant.

\section{Results}

\subsection{Patient Characteristics}

Eleven patients with definite gout had a mean age of 62.3 \pm 10.7 years and were predominantly male $(73 \%)$. The pseudogout patients $(\mathrm{n}=12)$ were significantly older, with a mean age of $78 \pm 9.3$ years and an equal male to female sex ratio (1:1) (Table 1). Body mass index (BMI) trended towards statistical significance between gout and pseudogout patients at $31.1 \pm 5.7 \mathrm{~kg} / \mathrm{m}^{2}$ and $25.4 \pm 6.0$ $\mathrm{kg} / \mathrm{m}^{2}$ respectively (Table 1). Serum uric acid levels were significantly higher in gout than in pseudogout patients, $11.4 \pm 3.5 \mathrm{mg} / \mathrm{dL}$ and $6.0 \pm 1.7 \mathrm{mg} / \mathrm{dL}$ respectively. The serum creatinine was higher in gout patients

Table 1. Gout and pseudogout patient demographics, serum uric acid, serum creatinine, peripheral white blood cell count and synovial fluid white cell count expressed as mean \pm SD (range) median, unless otherwise stated. * Significant at $P<0.05$.

\begin{tabular}{|c|c|c|c|}
\hline & Gout $(n=11)$ & Pseudogout $(n=12)$ & $P$-value \\
\hline Age (years) & $62.3 \pm 10.7(38-77) 67$ & $78 \pm 9.3(61-88) 78$ & $0.002^{*}$ \\
\hline Sex (male/female) & $8 / 3$ & $6 / 6$ & 0.292 \\
\hline BMI $\left(\mathrm{kg} / \mathrm{m}^{2}\right)$ & $31.1 \pm 5.7(23-41.4) 31.3$ & $25.4 \pm 6.0(16.7-35.9) 24.5$ & 0.056 \\
\hline Serum uric acid (mg/dL) & $11.4 \pm 3.5(6.2-16.9) 11.8$ & $6.0 \pm 1.7(2.9-8.3) 6.2$ & $0.001^{*}$ \\
\hline Serum creatinine $(\mathrm{mg} / \mathrm{dL})$ & $1.5 \pm 0.5(0.9-2.5) 1.4$ & $1.3 \pm 0.7(0.6-2.9) 1.2$ & 0.205 \\
\hline Peripheral wbc $(\mathrm{k} / \mu \mathrm{L})$ & $9.27 \pm 3.7(5.3-18.3) 7.6$ & $8.9 \pm 3.2(2.1-13.8) 9.3$ & 0.877 \\
\hline
\end{tabular}


but not significantly so. The peripheral white blood cell count (wbc) and synovial fluid wbc were not statistically different between gout and pseudogout patients (Table 1).

\subsection{Differences in ESR and CRP between Gout, Pseudogout and Crystal-Induced Arthritis with Non-Articular Infection (CIA)}

There was no significant difference between the ESR in acute gout $(68.2 \pm 49.9 \mathrm{~mm} / \mathrm{Hr})$ and pseudogout $(83.9 \pm$ $45.6 \mathrm{~mm} / \mathrm{Hr}$ ) (Table 2). However there was a significant difference between the ESR in gout and CIA (113.8 \pm $37.2 \mathrm{~mm} / \mathrm{Hr}$ ) (Table 2). By contrast the CRP levels in both gout $(10.1 \pm 7.9 \mathrm{mg} / \mathrm{dL})$ and pseudogout $(18.9 \pm 9.8$ $\mathrm{mg} / \mathrm{dL}$ ) were significantly different from the levels in CIA $(36.5 \pm 12.4 \mathrm{mg} / \mathrm{dL})$. There was also a significant difference in CRP between gout and pseudogout by pairwise comparison. The overall trend was for higher levels of CRP and ESR in pseudogout than in gout and for higher levels of both in CIA than in pseudogout (Figures 1(a) and (b) respectively). Of note a single female patient aged 65 years with both MSUM and CPPD crystals showed a peripheral wbc of $15.1 \mathrm{k} / \mu \mathrm{L}$, BMI of 21.9 $\mathrm{kg} / \mathrm{m}^{2}$, synovial wbc of 24,200 cells $/ \mu \mathrm{L}$, serum uric acid level of $11.4 \mathrm{mg} / \mathrm{dL}$, serum creatinine of $2.8 \mathrm{mg} / \mathrm{dL}, \mathrm{CRP}$ of $40 \mathrm{mg} / \mathrm{dL}$ and ESR of $115 \mathrm{~mm} / \mathrm{Hr}$. Joint aspirate showed the presence of both MSUM and CPPD crystals. There was radiographic evidence of chondrocalcinosis as well.

\subsection{Correlation of Peripheral wbc and Synovial wbc with ESR and CRP in Gout and Pseudogout}

There was no significant correlation between the ESR and the CRP in gout patients with the peripheral wbc or synovial wbc levels (Table 3). Neither did ESR correlate with CRP in pseudogout patients. There was a trend to statistical significance between the peripheral wbc in

Table 2. (a) Pair-wise comparison of means for ESR and CRP in A/D. gout, B/E. pseudogout and C/F. CIA patients using Mann-Whitney U-test. "Significant at $P<0.05$; (b) Comparison of group means by ANOVA. "Significant at $P<0.05$.

(a)

\begin{tabular}{|c|c|c|c|}
\hline ESR & \multicolumn{3}{|c|}{ P-value for pair-wise comparison of ESR means } \\
\hline & A. Gout ESR $68.2 \pm 49.9(5-122) 73$ & B. Pseudogout ESR $83.9 \pm 45.6(20-140) 88.5$ & C. CIA ESR $113.8 \pm 37.2(50-140) 124$ \\
\hline A vs B & & 0.230 & \\
\hline A vs C & & $0.041^{*}$ & \\
\hline B vs $\mathrm{C}$ & & 0.169 & \\
\hline CRP & \multicolumn{3}{|c|}{$P$-value for pair-wise comparison of CRP means } \\
\hline & D. Gout CRP $10.1 \pm 7.9(0.3-24.7) 8.7$ & E. Pseudogout CRP $18.9 \pm 9.8(5.8-32.8) 15.4$ & F. CIA CRP $36.5 \pm 12.4(16.7-45.2) 43.5$ \\
\hline D vs E & & $0.037^{*}$ & \\
\hline D vs F & & $0.003^{*}$ & \\
\hline E vs F & & $0.020^{*}$ & \\
\hline
\end{tabular}

(b)

\begin{tabular}{cc}
\hline & ANOVA $P$-value \\
\hline ESR & 0.2067 \\
CRP & $0.0001^{*}$ \\
\hline
\end{tabular}

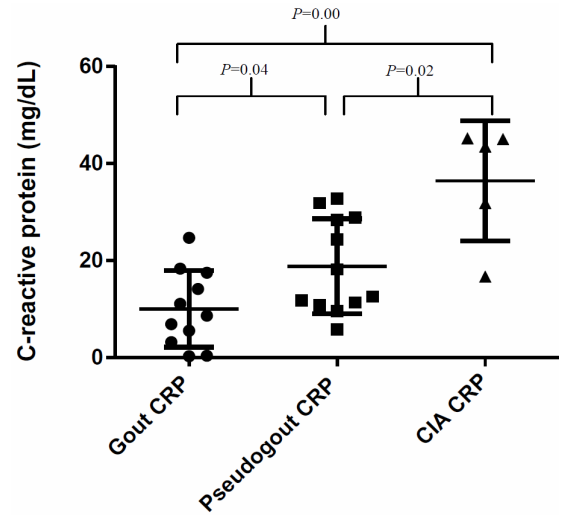

(a) CRP

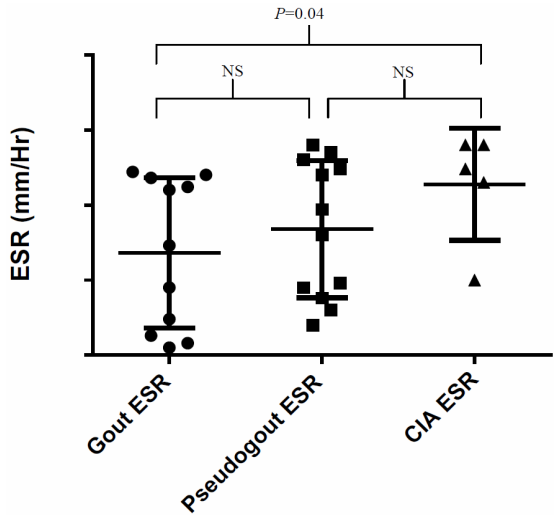

(b) ESR

Figure 1. Comparison of CRP and ESR between gout, pseudogout and CIA. Error bars represent mean \pm S.D. Statistical differences are summarized in Table 2 . NS = not significant. 
gout and the gout CRP with a $P$-value of 0.052 (Table 3). These results suggest that the peripheral and synovial cell counts are of little utility in differentiating between gout and pseudogout.

\subsection{Comparison of Peripheral wbc and Synovial wbc between Gout, Pseudogout and CIA}

There was a significant difference between the peripheral wbc in gout and CIA (Table 4). There was also a significant difference between the peripheral wbc in pseudogout and CIA (Table 4). However, there was no significant relationship between the levels of synovial wbc comparing gout and CIA, or between pseudogout and patients with CIA (Table 4). This suggests that marked elevations of the peripheral wbc are more suggestive of the presence of infection than of the existence of crystalinduced arthritis. It also suggests that unlike the case in septic arthritis the presence of infection at a non-articular site probably does not significantly influence the synovial wbc [11].

\section{Discussion}

We have examined the relationship between the acute phase response and the acute phase reactants, ESR and CRP in acute gout and pseudogout. We have compared those responses in patients with acute gouty arthritis or pseudogout in the presence or absence of non-intra-articular infections. Patients with gout were significantly younger than pseudogout patients, were more likely to be male and had higher levels of serum uric acid. There was a trend to higher BMI in the gout patients echoing findings observed in population studies [12]. The association of renal impairment with gout was reflected by the higher serum creatinine in the gout patients though this was not statistically significant perhaps because of the small sample size (Table 1). The congruence of these observations with reported findings suggests that the patient sample though small was fairly representative of the larger gout and pseudogout populations.

There was a significant difference between the levels of ESR in gout and CIA but not between the gout-ESR and pseudogout-ESR. However, there was a significant difference between the CRP in gout and pseudogout and more so between gout and CIA (Table 2). This incremental difference was similar to that seen with the ESR but additionally was statistically significant (Figure 1).

There were no positive clinical correlations between peripheral or synovial cell counts and ESR or CRP in gout and pseudogout (Table 3). By contrast there was a significant correlation between the peripheral wbc in both gout and pseudogout, and the peripheral wbc in patients with gout or pseudogout in the presence of an infection (Table 4). This suggests that in crystal-induced arthritis there is a rise in CRP that is not associated with a proportional rise in the peripheral wbc, i.e. there is a CRP-peripheral wbc dissociation in crystal-induced arthritis, particularly in pseudogout. In addition, the synovial wbc is only helpful when there is intra-articular infection and cannot distinguish between gout and pseudogout [11]. However the single patient with mixed crystal arthritis and a peripheral wbc of $15.1 \mathrm{k} / \mu \mathrm{L}$ might suggest that gout with pseudogout may be associated with significant peripheral wbc elevations. However the current study did not have enough patients to make a definitive determination and the finding calls for further study.

Table 3. Correlation of peripheral wbc and articular wbc with ESR and CRP in gout and pseudogout. Significant at $P<0.05$.

\begin{tabular}{ccc}
\hline Correlation & Spearman r & $P$ value \\
\hline Gout peripheral wbc with gout ESR & 0.207 & 0.514 \\
Gout peripheral wbc with gout CRP & 0.606 & 0.052 \\
Pseudogout peripheral wbc with pseudogout ESR & 0.364 & 0.246 \\
Pseudogout peripheral wbc with pseudogout CRP & -0.336 & 0.287 \\
Gout articular wbc with gout ESR & 0.335 & 0.385 \\
Gout articular wbc with gout CRP & 0.301 & 0.437 \\
Pseudogout articular wbc with pseudogout ESR & -0.210 & 0.514 \\
Pseudogout articular wbc with pseudogout CRP & 0.154 & 0.635 \\
\hline
\end{tabular}

Table 4. Comparison of gout and pseudogout peripheral wbc and articular wbc with peripheral wbc and articular wbc in CIA. * Significant at $P<0.05$.

\begin{tabular}{|c|c|c|c|c|}
\hline & Gout $(\mathrm{n}=11)$ & Pseudogout $(n=12)$ & CIA (Gout $n=3$, Pseudogout $n=2$ ) & $\begin{array}{c}P \text { values } \\
\text { (Gout vs CIA/Pseudogout vs CIA) }\end{array}$ \\
\hline Peripheral wbc $(\mathrm{k} / \mu \mathrm{L})$ & $9.27 \pm 3.7(5.3-18.3) 7.6$ & $8.9 \pm 3.2(2.1-13.8) 9.3$ & $16.5 \pm 6.8(9.1-27.1) 14.3$ & $0.023^{*} / 0.020^{*}$ \\
\hline Synovial wbc (cells $/ \mu \mathrm{L})$ & $\begin{array}{c}13,133 \pm 12,196 \\
(1100-41,550) 8800\end{array}$ & $\begin{array}{c}14,790 \pm 21,419 \\
(1225-80,000) 8150\end{array}$ & $\begin{array}{c}29,580 \pm 23,320 \\
(6700-67,200) 25,100\end{array}$ & $0.182 / 0.073$ \\
\hline Infection diagnoses & & & $\begin{array}{c}\text { Cellulitis } 3 \\
\text { Pneumonia } 1 \\
\text { Urinary tract infection } 1\end{array}$ & \\
\hline
\end{tabular}


Possible reasons for the differences in the acute phase response, ESR and CRP between acute gout and pseudogout could be explained by differences in their cytokine profiles. Several studies suggest that the release of interleukin-1 $\beta$ (IL-1 $\beta$ ) from monocytes is similar between gout and pseudogout, suggesting equal activation of the inflammasome $[13,14]$. However, significant differences may exist in the release of interleukin-6 (IL-6) as suggested by data from Guerne et al [15]. Release of IL-6 from monocytes stimulated in vitro with CPPD crystals was more than seen with MSUM crystals although a higher concentration of the former crystals was required for peak IL-6 levels. Hydroxyapatite crystals were the least vigorous in stimulating IL-6 secretion [15]. The authors also demonstrated IL-6 release from synoviocytes and showed that both MSUM and CPPD cause release of IL-6 into the synovial fluid. Work by Liu et al [16] suggested that there was differential release of interleukin-8 (IL-8) by monocytes stimulated with MSUM or CPPD crystals with the latter causing significantly more IL- 8 release. The clinical relevance of IL- 8 may be in the massive cellular recruitment that characterizes pseudogout, which sometimes presents with the pseudoseptic picture. It is not known if there are differences in the release by monocytes and other cell types of interleukin- $1 \alpha$ upon stimulation by microcrystals although such inflammasome-dependent release has been documented [17]. Other cytokines like tumor necrosis factor- $\alpha$ (TNF- $\alpha$ ) have been implicated in the inflammatory response of monocytes and synoviocytes to microcrystals [18]. Microcrystals are known to induce the differentiation of monocytes to the active M1 phenotype that are involved in the release of inflammatory cytokines [19].

$\mathrm{C}$-reactive protein is produced predominantly by hepatocytes under the influence of IL-1 $\beta$ and IL-6 [20]. Since the release of IL- 6 is not inflammasome dependent we speculate that IL- $1 \beta$ production leads to IL- 6 release in crystal-induced arthritis as a downstream event following inflammasome activation $[19,21,22]$. Various cell types have inflammasomes including neutrophils, monocytes, macrophages and synovial fibroblast-like cells and are probably involved in the recognition of microcrystals [23]. The higher levels of CRP in pseudogout could reflect higher release of IL-6 in pseudogout, acting in concert with IL-1 $\beta$ on hepatocytes. Indeed Desgeorges et al. [22] demonstrated that patients with chondrocalcinosis had higher levels of both synovial fluid IL-6 and soluble IL-6 receptor- $\alpha$ than osteoarthritis patients and had levels comparable to patients with gout or rheumatoid arthritis. Serum levels of those molecules were also elevated [22]. The study did not specify whether chondrocalcinosis patients had active pseudogout at the time of joint aspiration, or osteoarthritis with the isolation of CPPD crystals from joint aspirates. Therefore it is difficult to conclude where on the CPPD disease spectrum they were. However, one could speculate that IL-6 release may predominate in pseudogout causing the differential rise in CRP. This could be due in part to the negative regulatory influence of IL-6 in gout [24]. It could also be due to the modulation of transforming growth factor- $\beta$ (TGF- $\beta$ ) and other anti-inflammatory cytokines produced by M2 macrophages since TGF- $\beta$ may have a permissive role in pseudogout and CPPD disease distinct from its purely antiinflammatory role in gout $[25,26]$. This could lead to a prolongation of the proinflammatory influence of IL-6 in pseudogout.

Regardless of the precise mechanism by which CRP is increased in pseudogout, it is possible that its release is augmented in the presence of mixed microcrystals. One patient had both MSUM and CPPD crystals with a significant elevation of CRP of $40 \mathrm{mg} / \mathrm{dL}$. However the single patient did not allow for any definite conclusions and further studies are needed. In conclusion, our study suggests differences in the acute phase response between gout and pseudogout, which might aid in diagnosis. The absence of similar findings by Söderquist et al. may be explained by their failure to separate gout from pseudogout [27]. We think that marked elevations of peripheral wbc should alert the clinician to the presence of infection. We also believe that significant elevations in CRP particularly in the absence of corresponding elevations in peripheral wbc should suggest pseudogout over gouty arthritis. Lastly we would recommend extreme vigilance for mixed microcrystal deposits or the presence of sepsis in patients with both marked elevations in peripheral wbc and CRP. Marked elevations in synovial wbc remain the most useful marker of septic arthritis [11].

\section{Study limitations}

The study is limited by the small sample size and therefore might not be representative of the larger population. In addition, the study was retrospective. However, the small size may have increased the threshold for statistical significance and suggest that the positive findings may hold up in a larger study. However, these findings will need to be verified in a larger prospectively designed study.

\section{REFERENCES}

[1] C. M. Wise, "Crystal-Associated Arthritis in the Elderly," Rheumatic Disease Clinics of North America, Vol. 33, No. 1, 2007, pp. 33-55. http://dx.doi.org/10.1016/j.rdc.2006.12.007

[2] A. J. Elliot, K. W. Cross and D. M. Fleming, "Seasonality and Trends in the Incidence and Prevalence of Gout in England and Wales 1994-2007," Annals of the Rheumatic 
Diseases, Vol. 68, No. 11, 2009, pp. 1728-1733. http://dx.doi.org/10.1136/ard.2008.096693

[3] P. Richette, T. Bardin and M. Doherty, "An Update on the Epidemiology of Calcium Pyrophosphate Dihydrate Crystal Deposition Disease," Rheumatology (Oxford), Vol. 48, No. 7, 2009, pp. 711-715.

http://dx.doi.org/10.1093/rheumatology/kep081

[4] E. Roddy and M. Doherty, "Epidemiology of Gout," Arthritis Research \& Therapy, Vol. 12, 2010 p. 223. http://dx.doi.org/10.1186/ar3199

[5] Y. Zhu, B. J. Pandya and H. K. Choi, "Prevalence of Gout and Hyperuricemia in the US General Population: The National Health and Nutrition Examination Survey 2007-2008," Arthritis \& Rheumatism, Vol. 63, No. 10, 2011, pp. 3136-3141. http://dx.doi.org/10.1002/art.30520

[6] L. Trieste, I. Palla, F. Fusco, et al., "The Economic Impact of Gout: A Systematic Literature Review," Clinical and Experimental Rheumatology, Vol. 30, No. 4, 2012, pp. S145-S148.

[7] N. Busso and A. So, "Microcrystals as DAMPs and Their Role in Joint Inflammation," Rheumatology (Oxford), Vol. 51, No. 7, 2012, pp. 1154-1160. http://dx.doi.org/10.1093/rheumatology/ker524

[8] S. L. Wallace, H. Robinson, A. T. Masi, J. L. Decker, D. J. McCarty and T. F. Yu, "Preliminary Criteria for the Classification of the Acute Arthritis of Primary Gout," Arthritis \& Rheumatism, Vol. 20, No. 3, 1977, pp. 895900. http://dx.doi.org/10.1002/art.1780200320

[9] D. J. McCarty, "Calcium Pyrophosphate Dihydrate Crystal Deposition Disease: Nomenclature and Diagnostic Criteria," Annals of Internal Medicine, Vol. 87, No. 2, 1977, pp. 241-242.

http://dx.doi.org/10.7326/0003-4819-87-2-240

[10] W. Zhang, M. Doherty, T. Bardin, et al., "European League against Rheumatism Recommendations for Calcium Pyrophosphate Deposition. Part I: Terminology and Diagnosis," Annals of the Rheumatic Diseases, Vol. 70, No. 4, 2011, pp. 563-570. http://dx.doi.org/10.1136/ard.2010.139105

[11] M. E. Margaretten, J. Kohlwes, D. Moore and S. Bent, "Does This Adult Patient Have Septic Arthritis?" JAMA, Vol. 297, No. 13, 2007, pp. 1478-1488. http://dx.doi.org/10.1001/jama.297.13.1478

[12] T. Neogi, "Clinical Practice. Gout," New England Journal of Medicine, Vol. 364, No. 5, 2011, pp. 443-452. http://dx.doi.org/10.1056/NEJMcp1001124

[13] F. Martinon, V. Petrilli, A. Mayor, A. Tardivel and J. Tschopp, "Gout-Associated Uric Acid Crystals Activate the NALP3 Inflammasome," Nature, Vol. 440, No. 7081, 2006, pp. 237-241. http://dx.doi.org/10.1038/nature04516

[14] R. Liu-Bryan and F. Liote, "Monosodium Urate and Calcium Pyrophosphate Dihydrate (CPPD) Crystals, Inflammation, and Cellular Signaling," Joint Bone Spine, Vol. 72, No. 3, 2005, pp. 295-302. http://dx.doi.org/10.1016/j.jbspin.2004.12.010

[15] P. A. Guerne, R. Terkeltaub, B. Zuraw and M. Lotz, "Inflammatory Microcrystals Stimulate Interleukin-6 Production and Secretion by Human Monocytes and Syno- viocytes," Arthritis \& Rheumatism, Vol. 32, No. 11, 1989 , pp. 1443-1452. http://dx.doi.org/10.1002/anr.1780321114

[16] R. Liu, M. O’Connell, K. Johnson, K. Pritzker, N. Mackman and R. Terkeltaub, "Extracellular Signal-Regulated Kinase 1/Extracellular Signal-Regulated Kinase 2 Mitogen-Activated Protein Kinase Signaling and Activation of Activator Protein 1 and Nuclear Factor KappaB Transcription Factors Play Central Roles in Interleukin-8 Expression Stimulated by Monosodium Urate Monohydrate and Calcium Pyrophosphate Crystals in Monocytic Cells," Arthritis \& Rheumatism, Vol. 43, No. 5, 2000, pp. 1145-1155.

http://dx.doi.org/10.1002/1529-0131(200005)43:5<1145:: AID-ANR25>3.0.CO;2-T

[17] O. Gross, A. S. Yazdi, C. J. Thomas, et al., "Inflammasome Activators Induce Interleukin-1Alpha Secretion via Distinct Pathways with Differential Requirement for the Protease Function of Caspase-1," Immunity, Vol. 36, No. 3, 2012, pp. 388-400.

http://dx.doi.org/10.1016/j.immuni.2012.01.018

[18] F. S. di Giovine, S. E. Malawista, E. Thornton and G. W. Duff, "Urate Crystals Stimulate Production of Tumor Necrosis Factor Alpha from Human Blood Monocytes and Synovial Cells. Cytokine mRNA and Protein Kinetics, and Cellular Distribution," The Journal of Clinical Investigation, Vol. 87, No. 4, 1991, pp. 1375-1381. http://dx.doi.org/10.1172/JCI115142

[19] W. J. Martin, O. Shaw, X. Liu, S. Steiger and J. L. Harper, "Monosodium Urate Monohydrate Crystal-Recruited Noninflammatory Monocytes Differentiate into M1-Like Proinflammatory Macrophages in a Peritoneal Murine Model of Gout," Arthritis \& Rheumatism, Vol. 63, No. 5, 2011, pp. 1322-1332. http://dx.doi.org/10.1002/art.30249

[20] A. W. Taylor, N. O. Ku and R. F. Mortensen, "Regulation of Cytokine-Induced Human C-Reactive Protein Production by Transforming Growth Factor-Beta," The Journal of Immunology, Vol. 145, No. 8, 1990, pp. 2507-2513.

[21] G. Tosato and K. D. Jones, "Interleukin-1 Induces Interleukin-6 Production in Peripheral Blood Monocytes," Blood, Vol. 75, No. 6, 1990, pp. 1305-1310.

[22] A. Desgeorges, C. Gabay, P. Silacci, et al., "Concentrations and Origins of Soluble Interleukin 6 Receptor-Alpha in Serum and Synovial Fluid," The Journal of Rheumatology, Vol. 24, No. 8, 1997, pp. 1510-1516.

[23] K. Schroder and J. Tschopp, "The Inflammasomes," Cell, Vol. 140, No. 6, 2010, pp. 821-832. http://dx.doi.org/10.1016/j.cell.2010.01.040

[24] H. Tsutani, N. Yoshio and T. Ueda, "Interleukin 6 Reduces Serum Urate Concentrations," The Journal of Rheumatology, Vol. 27, No. 2, 2000, p. 554.

[25] N. Busso and H. K. Ea, "The Mechanisms of Inflammation in Gout and Pseudogout (CPP-Induced Arthritis)," Reumatismo, Vol. 63, No. 4, 2011, pp. 230-237.

[26] L. Punzi, F. Oliviero and R. Ramonda, "Transforming Growth Factor-Beta Levels in Synovial Fluid of Osteoarthritis with or without Calcium Pyrophosphate Dihydrate Crystals," The Journal of Rheumatology, Vol. 30, No. 2, 2003, p. 420. 
[27] B. Söderquist, I. Jones, H. Fredlund and T. Vikerfors, "Bacterial or Crystal-Associated Arthritis? Discriminating Ability of Serum Inflammatory Markers," Scandinavian
Journal of Infectious Diseases, Vol. 30, No. 6, 1998, pp. 591-596. http://dx.doi.org/10.1080/00365549850161151 